\title{
The transcription factor Uncx4.1 acts in a short window of midbrain dopaminergic neuron differentiation
}

\author{
Tamara I Rabe ${ }^{1,2}$, Gundula Griesel1 ${ }^{1}$, Stephen Blanke ${ }^{1}$, Andreas Kispert ${ }^{3}$, Michael Leitges ${ }^{4}$, Bert van der Zwaag ${ }^{5,8}$, \\ J Peter H Burbach ${ }^{5}$, Frédérique Varoqueaux ${ }^{6}$ and Ahmed Mansouri ${ }^{1,2,7^{*}}$
}

\begin{abstract}
Background: The homeobox containing transcription factor Uncx4.1 is, amongst others, expressed in the mouse midbrain. The early expression of this transcription factor in the mouse, as well as in the chick midbrain, points to a conserved function of Uncx4.1, but so far a functional analysis in this brain territory is missing. The goal of the current study was to analyze in which midbrain neuronal subgroups Uncx4.1 is expressed and to examine whether this factor plays a role in the early development of these neuronal subgroups.

Results: We have shown that Uncx4.1 is expressed in GABAergic, glutamatergic and dopaminergic neurons in the mouse midbrain. In midbrain dopaminergic (mDA) neurons Uncx4.1 expression is particularly high around E11.5 and strongly diminished already at E17.5. The analysis of knockout mice revealed that the loss of Uncx4.1 is accompanied with a $25 \%$ decrease in the population of mDA neurons, as marked by tyrosine hydroxylase (TH), dopamine transporter (DAT), Pitx3 and Ngn2. In contrast, the number of glutamatergic Pax6-positive cells was augmented, while the GABAergic neuron population appears not affected in Uncx4.1-deficient embryos.

Conclusion: We conclude that Uncx4.1 is implicated in the development of mDA neurons where it displays a unique temporal expression profile in the early postmitotic stage. Our data indicate that the mechanism underlying the role of Uncx4.1 in mDA development is likely related to differentiation processes in postmitotic stages, and where Ngn2 is engaged. Moreover, Uncx4.1 might play an important role during glutamatergic neuronal differentiation in the mouse midbrain.
\end{abstract}

Keywords: Uncx4.1, Midbrain, mDA neurons, Ngn2, Pax6, Differentiation, Expression

\section{Background}

Midbrain dopaminergic (mDA) neurons are organized in three different areas in the mammalian midbrain: the substantia nigra pars compacta ( $\mathrm{SNpc}$ ), the ventral tegmental area (VTA), and the retrorubal field (RRF). mDA neurons from the SNpc are associated with the nigrostriatal pathway while neurons from the VTA and RRF act in the mesolimbic pathway. The degeneration of neurons of the nigrostriatal pathway in Parkinson's disease has attracted many researchers to identify and study

\footnotetext{
* Correspondence: amansou@gwdg.de

'Department of Molecular Cell Biology, Max Planck Institute of Biophysical

Chemistry, Am Fassberg 11, Goettingen 37077, Germany

${ }^{2}$ DFG Research Center for the Molecular Physiology of the Brain, CMPB,

Humboldtallee 23, Goettingen 37073, Germany

Full list of author information is available at the end of the article
}

the transcriptional network controlling $\mathrm{mDA}$ neuron development. Although several factors and determinants were found to be crucial for mDA neuron development like Ngn2 [1,2], Lmx1a/1b [3], Foxa1/2 [4,5], Nurr1 [6], Otx2 [7] and $\beta$-Catenin $[8,9]$ the molecular mechanisms controlling specification and differentiation of these neurons are not fully understood, and the knowledge of the transcriptional network is still incomplete.

Uncx4.1 is a homeobox containing transcription factor with $88 \%$ identity to Unc4 from C.elegans $[10,11]$. In mice, it is expressed in the caudal half of the somite, the developing kidney, and the central nervous system [10,11]. Functional analysis using knockout mice indicated that Uncx4.1 is required for the proper development of the axial skeleton $[12,13]$. In the central nervous system

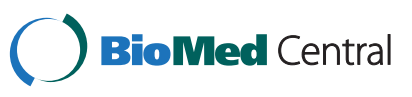


Uncx4.1 is detected in the spinal cord, the mesencephalon and the telencephalon $[10,11]$. Recent findings provided evidence for the involvement of Uncx4.1 in late development of the pituitary neural lobe [14], and the proliferation of neural progenitors, as well as neuronal survival in the mouse olfactory epithelium [15]. In contrast, the possible role of Uncx4.1 in the developing midbrain has not been addressed.

Here we show that in the ventral midbrain Uncx4.1 is found to localize with dopaminergic, glutamatergic and GABAergic postmitotic neurons at embryonic day (E) 11.5 of gestation. Molecular analysis of global loss-of-function mouse mutant revealed that $\mathrm{mDA}$ neurons are reduced in the absence of Uncx4.1, and this is corroborated by a partial downregulation of $N g n 2$. Interestingly the number of glutamatergic Pax6-expressing cells in the midbrain is increased at E13.5 while the Nkx6.1-positive neurons are not affected.

\section{Results}

Uncx4.1 is expressed in the mantle layer of the developing midbrain

In the central nervous system Uncx4.1 transcripts first appeared at E9.5 in the mesencephalon [10,13], but a detailed expression analysis of Uncx4.1 in the developing mouse midbrain at subsequent stages so far has not been performed. At E10.5, transcripts were detected in the mantle layer of the basal and alar plate (data not shown). At E11.5 the signal in the basal and alar plate was increased and Uncx4.1 protein was detected in the ventral-most midbrain, close to the mesencephalic flexure (Figure 1B-C, Figure 2A and data not shown). At E13.5 Unc 4.1 expression was scattered and the strongest labeling was observed in the lateral part of the basal plate (Figure 1D). At E17.5, Uncx4.1 expression remained scattered and the transcripts were distributed over the whole midbrain (Figure 1E). At all analyzed stages the expression

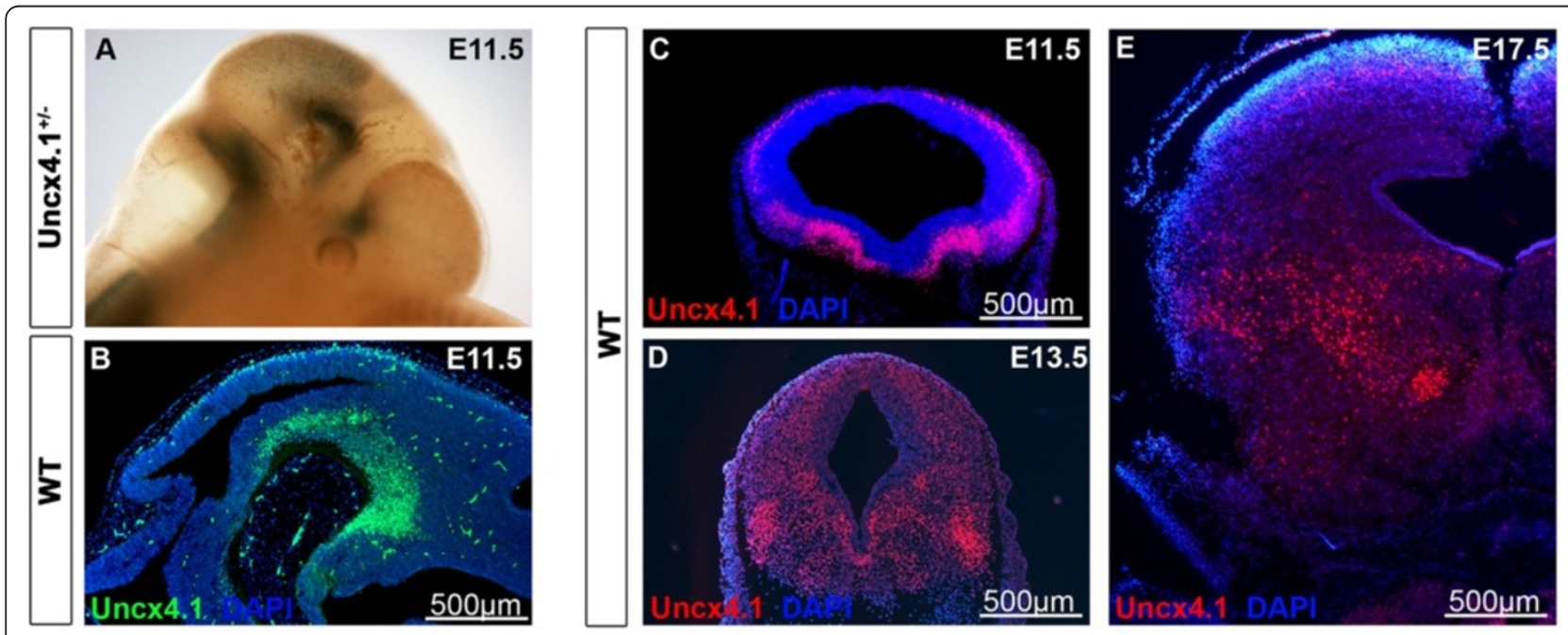

Uncx4.1 Tuj1 DAPI
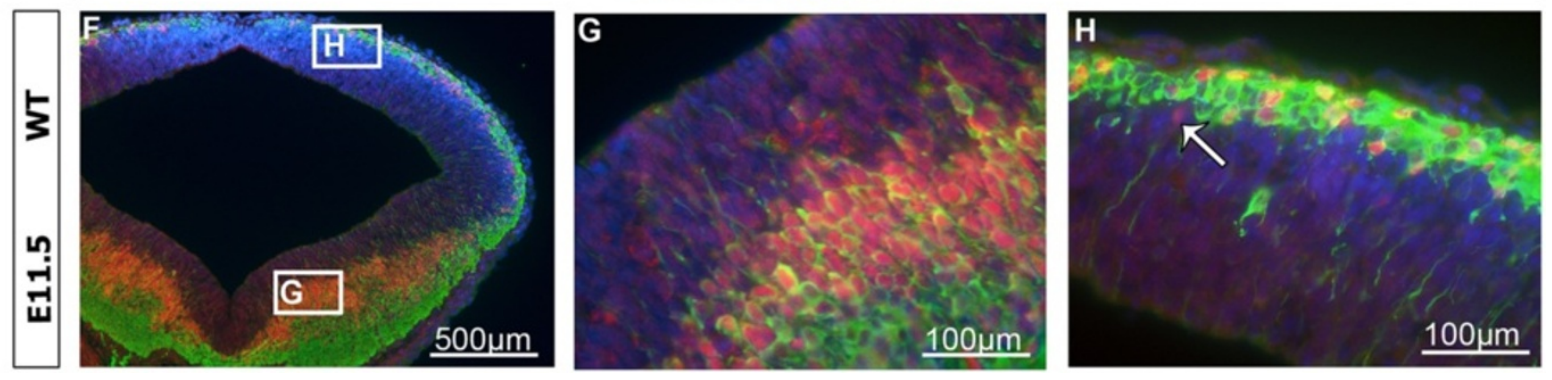

Figure 1 The expression of Uncx4.1 in the developing mouse midbrain. (A) X-gal staining of Uncx4.1 $1^{+/-}$embryo at E11.5.

(B-E) Immunohistochemistry (IHC) on coronal (D, C, E) and sagittal (B) sections of wild-type embryos showing the expression of Uncx4.1 in the developing midbrain at E11.5 (B-C), E13.5 (D) and E17.5 (E). (B, C) At E11.5 Uncx4.1 is expressed in the whole mantle layer of the embryonic midbrain. (D, E) At E13.5 and E17.5 Uncx4.1 expression appears in a salt-and-pepper pattern with Uncx4.1-negative cells. The strongest Uncx4.1 expression can be detected in the basal plate. (F-H) Double IHC with anti-Uncx4.1 and anti-Tuj1 on coronal sections of a wild-type embryo at E11.5. (G, H) Showing higher magnifications of F, referring to the labeling in the white box. The white arrow marks an Uncx4.1-positve, Tju1-negative cell. E, embryonic day. 


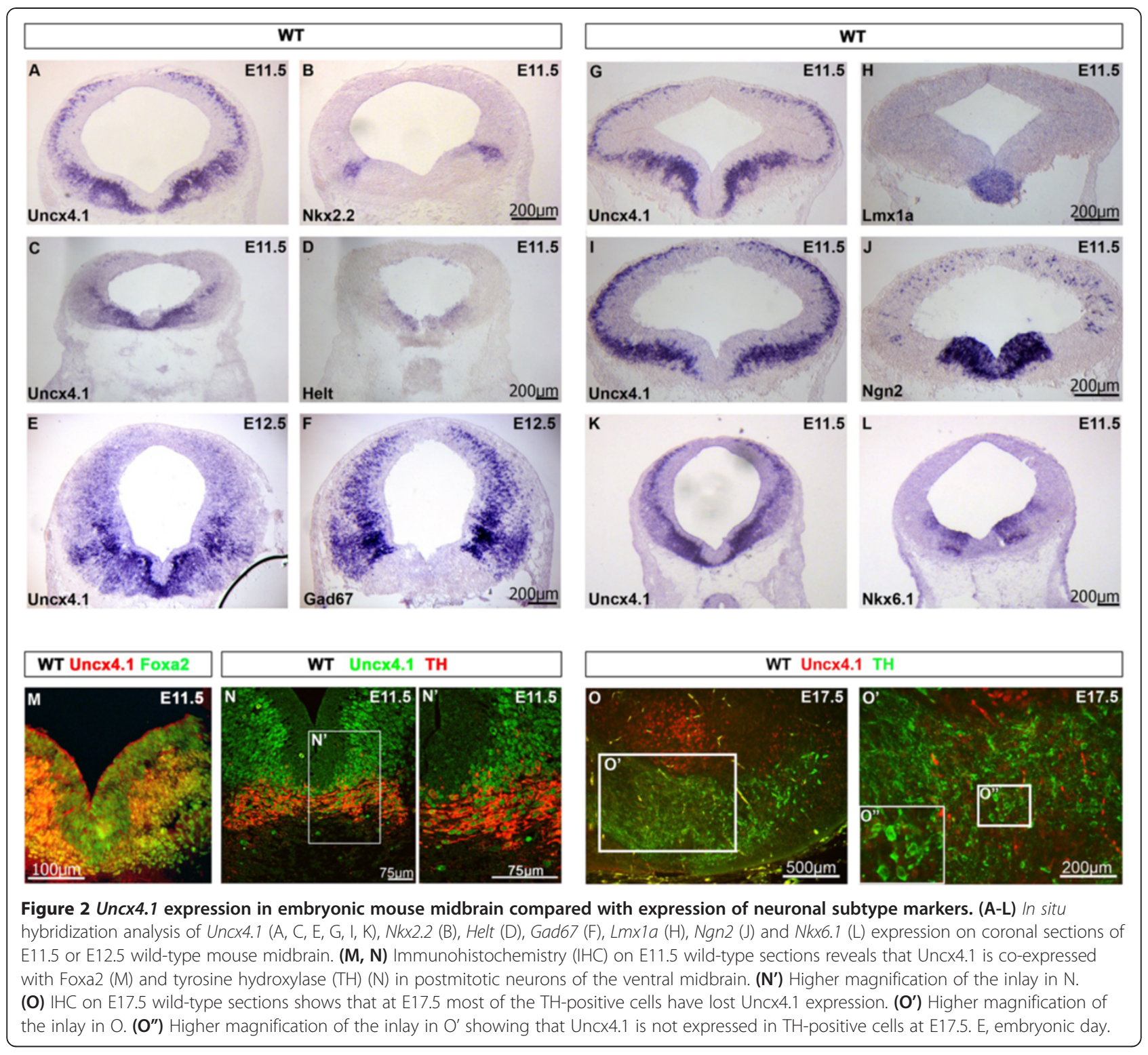

of Uncx4.1 was similar throughout the rostral-caudal axis of the midbrain and it was excluded from the midbrain ventricular zone $(\mathrm{VZ})$, suggesting that it is confined to postmitotic cells.

Double immunohistochemistry (IHC) with Uncx4.1 and Tju1, a postmitotic neuron marker, revealed that nearly all Uncx4.1-positive cells in the mantle layer also express Tuj1 (Figure 1F-H). Only in the dorsal midbrain a few Uncx4.1-positive and Tju1-negative cells could be detected (Figure $1 \mathrm{H}$ ). To examine which neuronal subtypes are marked by Uncx4.1 we compared the expression pattern of Uncx4.1 and the neuronal subtype markers: Lmx1a (mDA neurons), Gad67, Nkx2.2, Helt (GABAergic neurons), $N k x 6.1$ (glutamatergic neurons) and $N g n 2$ (mDA and glutamatergic neurons). Shown in consecutive sections Uncx4.1 expression domain appeared to co-localize at least partially with markers for most neuronal subgroups (Figure 2). This is true for Gad67, a general marker for GABAergic neurons in the mantle layer of the whole midbrain (Figure 2E-F), as well as for $N k x 2.2$ (Figure 2A-B). As expected, Helt, which is expressed in the ventricular zone of GABAergic neurons [16,17], did not show overlapping expression with Uncx4.1 (Figure 2C-D), confirming that Uncx4.1 is absent from mesencephalic GABAergic progenitors. $N k x 6.1$ labels a specific subset of glutamatergic neurons in the ventricular zone and the mantle layer $[17,18]$. The partially overlapping expression domains of $N k x 6.1$ and Uncx4.1 in the mantle layer indicate that Uncx4.1 is expressed in midbrain glutamatergic neurons. In the most central area of the medial ventral midbrain region, where mature mDA neurons arise, Uncx4.1 expression shared 
some expression domains with dopamine (DA) markers, namely Lmx1a [3] and Ngn2 [1,2] (Figure 2G-H, I-J). In order to examine whether Uncx4.1 is expressed in GABAergic, glutamatergic and DA neurons we performed IHC of Uncx4.1 together with tyrosine hydroxylase $(\mathrm{TH})$ or Foxa2. The dopaminergic neuron marker $\mathrm{TH}$ started to be expressed around E11.5 in mature mDA neurons. At this stage, most TH-positive neurons expressed Uncx4.1 (Figure $2 \mathrm{~N}$ ), while this was not the case at E17.5 (Figure 2O, O'), where Uncx4.1 is downregulated in the VTA as well as SN. This finding suggests that Uncx4.1 labels the majority of mature mDA neurons at early development, but is rapidly downregulated when matured. Co-localization of Foxa2 and Uncx4.1 was observed in the whole mantle layer within the expression domain of Foxa2, implying that Uncx4.1 is expressed in $\mathrm{mDA}, \mathrm{GABAergic}$ and glutamatergic neurons. Taken together, our data indicate that Uncx4.1 expression is not confined to one neuronal lineage of mesencephalic neurons. Rather, Uncx4.1 is expressed in most subtypes of midbrain neurons, but is excluded from their proliferating progenitors. These data suggest that Uncx4.1 is engaged in processes of postmitotic differentiation.

\section{Loss of Uncx4.1 causes reduction of $\mathrm{mDA}$ neurons in the ventral midbrain}

To examine the role of Uncx4.1 in mDA development, we analyzed the expression of the dopamine transporter (DAT) and TH in Uncx4.1-deficient embryos at E11.5 and E17.5. We observed a clear reduction of DAT expression at E17.5 (Figure 3E-F). Therefore, the number of mDA neurons was assessed. Although the distribution of THpositive cells was normal in control and Uncx4.1 mutant embryos, the number of $\mathrm{TH}$-positive cells was found significantly reduced (Figure 3A-D, Q). Interestingly, the decrease of TH-positive cells at E11.5 was similar to the reduction observed at E17.5 (both $-26 \%$, Figure 3Q). The partial loss of mDA neurons was further confirmed by IHC for Pitx3, Nurr1, Calretinin and Calbindin. Pitx3 specifically labels mDA neurons [19-22] and the reduction of Pitx3-positive cells (-31\%), correlated with the diminution of TH-positive cells (Figure 3I-J, R). A similar observation was made for the Nurr1-labeled cells at E16.5 (reduction of 24\%). The number of Calbindin- and Calretinin-marked cells, which are both expressed in a subset of mDA neurons $[23,24]$, was drastically decreased in Uncx4.1 $1^{-1-}$ embryos $(-43 \%$ and $-58 \%$, respectively, Figure $3 \mathrm{M}-\mathrm{P}, \mathrm{R})$. The early onset in the reduction of $\mathrm{TH}$-positive cells in the ventral midbrain and the corresponding downregulation of all tested markers indicates that Uncx4.1 plays an early role during development of mDA neurons. Furthermore, the dramatic reduction of Calbindin in the ventral midbrain suggests that the mDA neurons of the VTA are more affected as compared to the $\mathrm{SN}$, since Calbindin is preferentially expressed by VTA neurons [24]. To confirm the findings that the mDA neurons of the VTA are strongly affected in the absence of Uncx4.1, we analyzed the expression of Otx2 at E17.5 in Uncx4.1-deficient embryos. Otx2 is a transcription factor, which selectively labels postmitotic neurons of the VTA but not of the SN at E17.5, and it is expressed in a subset of Calbindin-positive neurons in this brain area [25]. Accordingly, Otx2 was downregulated in the VTA, confirming the findings from the Calbindin and Calretinin staining (Figure 3O-P). The partial depletion of $\mathrm{mDA}$ neurons may be a consequence of impaired cell proliferation or survival defects. To examine both possibilities we used 5-bromo-2'-deoxyuridine (BrdU) labeling for proliferation analysis and the terminal deoxynucleotidyl transferase dUTP nick end labeling (TUNEL) assay for investigating apoptosis. At E11.5, 24 hours after BrdU injection, as well as at E14.5, 45 minutes following BrdU injection, no alterations in numbers of BrdU-positive cells were observed (Additional file 1: Figure S1 A-B and data not shown). To further confirm that the decreased number of mDA neurons is not caused by alterations in cell proliferation of this neuronal subgroup, we analyzed the BrdUlabeled cells together with TH at E11.5, 24 hours following BrdU-injection. In the wild-type (WT) as well as in mutant embryos, we could not detect any difference in cell proliferation (Additional file 1: Figure S1 C-D). Apoptotic cells were also not detected at E11.5, and E17.5 (data not shown). We concluded that defects in cell proliferation or apoptosis are not involved in the DA neuron loss provoked by Uncx 4.1 depletion.

\section{The expression of $\mathrm{Ngn} 2$ is downregulated in Uncx4.1-deficient embryos}

Since it appeared that $\mathrm{mDA}$ neuron reduction was not caused by impaired proliferation or cell death, we next investigated which factors may be affected by the absence of Uncx4.1, provoking the partial depletion of mDA neurons. Since $L m x 1 a, L m x 1 b$, and Ngn2 are involved for proper establishment of mDA neurons [1$3,26,27]$, we analyzed their expression in E11.0 ( $\operatorname{m} m \times 1 a)$, E11.5 (Ngn2) or E12.5 (Lmx1a and Lmx1b) Uncx4.1 (-/- $^{-1}$ embryos. As expected, $L m x 1 a$ and $L m x 1 b$ expression was normal in Uncx4.1-deficient embryos (Figure 4A-B, $\mathrm{M}-\mathrm{N}$ and data not shown) but surprisingly the level of Ngn 2 mRNA was reduced (Figure 4, C-F). Interestingly, the downregulation of $N g n 2$ transcripts was also observed in the dorsal part of the midbrain (Figure 4, EF). Nurr1 expression was found nearly lacking in Ngn2 mutant mice at E11.5, but Nurr1-positve cells were rescued at later stages of development [1]. We therefore investigated the expression of Nurr1 in the developing Uncx4.1 mutants and observed at E11.5 a 28\% loss of Nurr1-positive cells compared to control embryos 


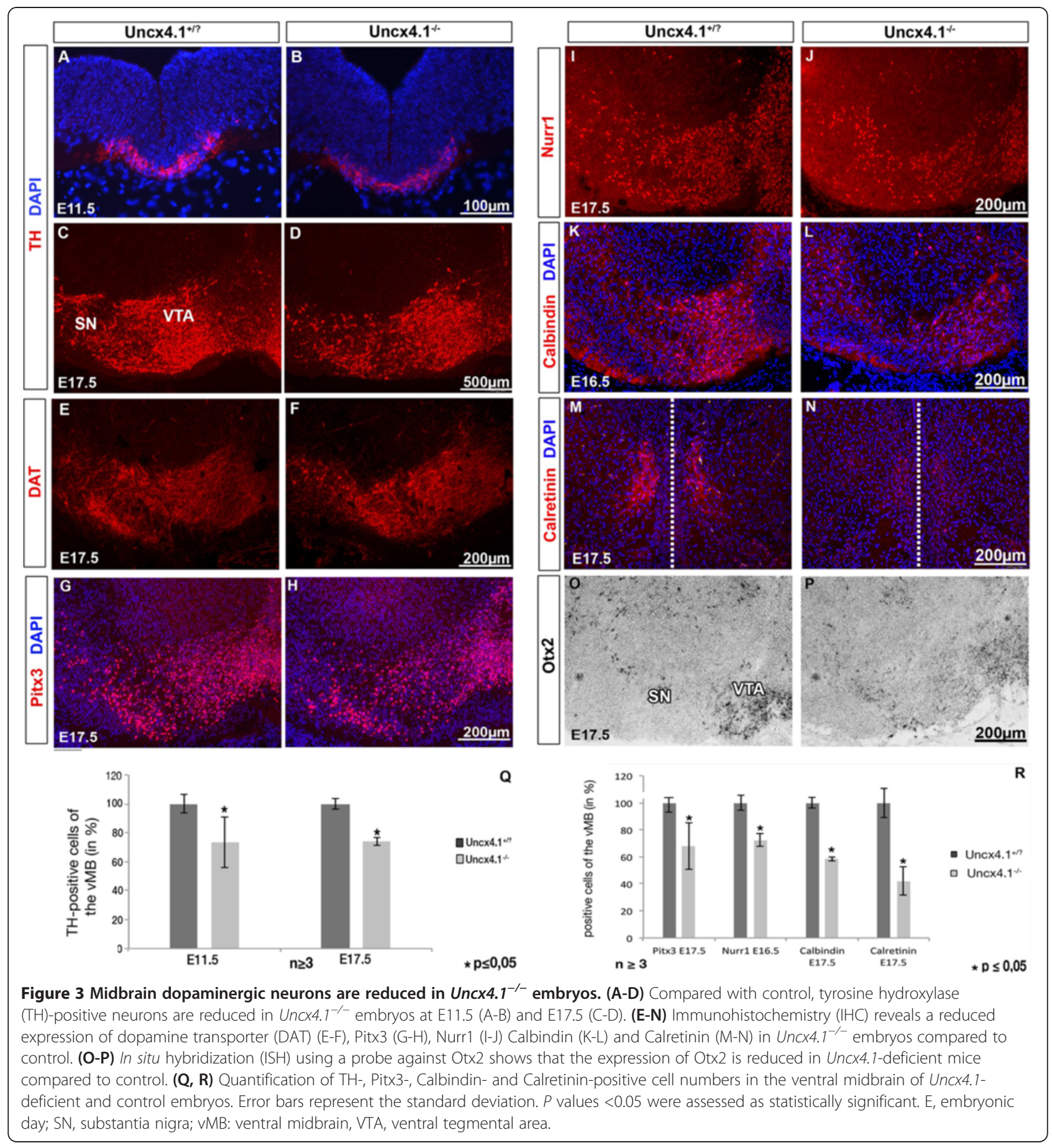

(Figure 4, I-J, O). A similar reduction of $23 \%$ occurred in E16.5 embryos (Figure 4, K-L, O). It has been previously speculated that the rescue of Nurr1-positive cells in $\mathrm{Ngn} 2^{-/-}$embryos is due to a compensation by the proneuronal gene Mash1 [1]. Its expression was completely missing at the ventral midline in E11.5 $\mathrm{Ngn}^{-/-}$embryos and was recovered at E13.5 [1]. In Uncx4.1-deficient midbrain the expression of Mash1 was found to be normal at E11.5 (Figure 4, G-H), indicating that unlike in $N g n 2^{-/-}$embryos, Nurr1-positive cells do not recover in the absence of Uncx4.1. Since neurogenin 2 (Ngn2) is required for the proper generation of $\mathrm{mDA}$ neurons, these results indicate that the alteration of $\mathrm{Ngn} 2$ expression may be responsible for $\mathrm{mDA}$ neuron reduction in the absence of Uncx4.1. We therefore analyzed the expression of Uncx4.1 and Ngn2 in more detail. Ngn2 


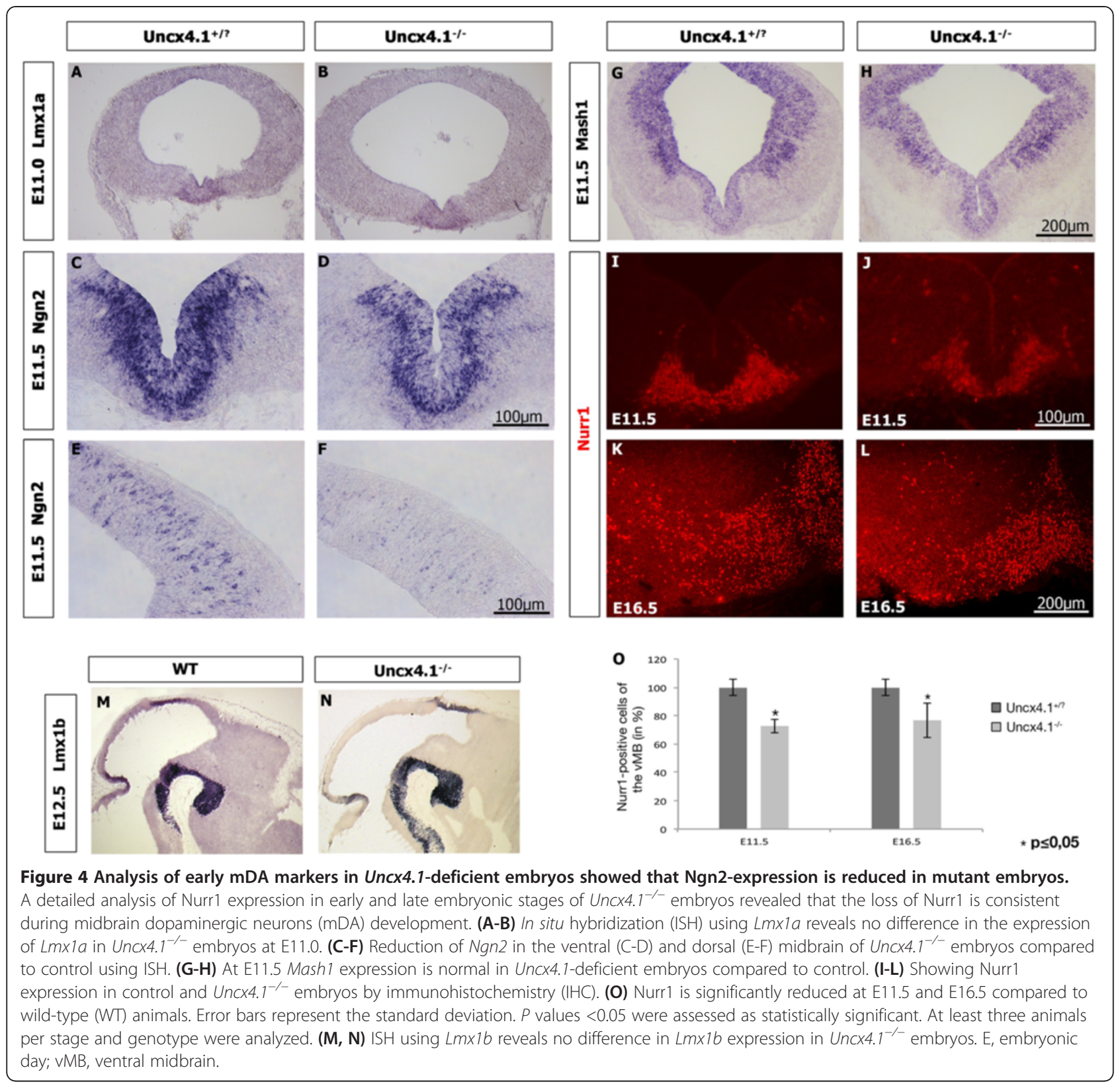

starts to be expressed in $\mathrm{mDA}$ progenitors and immature mDA neurons around E10.75 [1]. At E11.5 Ngn2 and Uncx4.1 co-positive cells were detected in the mantle layer of the ventral midbrain (Figure 5A-B, B'). It appears that on the mRNA level more double-positive cells were detected. The expression of Unxc4.1 in the ventral midbrain of $\mathrm{Ngn}^{-/-}$embryos was absent in the most ventral part of the midbrain at E11.5 and E13.5 (Figure 5C-F), where mDA neurons are located. This is consistent with the finding that Ngn2 is needed for the generation of postmitotic $\mathrm{mDA}$ precursors and that the progenitors are arrested in the absence of Ngn2 at E11.5 [1]. In contrast, Uncx4.1 expression in dorsal midbrain was not affected (data not shown). To further analyze whether Uncx4.1 and Ngn2 interact with each other, we performed a co-immunoprecipitation assay. Our results show that there is no interaction between Ngn2 and Uncx4.1 (Additional file 2: Figure S2), suggesting that these proteins do not undergo such a protein-protein interaction. Uncx4.1 may still interact with Ngn2 promoter. However, the palindromic putative binding site for Uncx4.1 (TAATYNRATTA) could not be identified on the Ngn2 locus. Taken together our findings suggest that the partial depletion of $\mathrm{mDA}$ neurons in Uncx4.1-deficient embryos may be a consequence of Ngn2 downregulation during an early phase of $\mathrm{mDA}$ neuron differentiation. How Uncx4.1 regulates Ngn2 expression remains elusive. It is possible that the observed downregulation of $\mathrm{Ngn} 2$ 


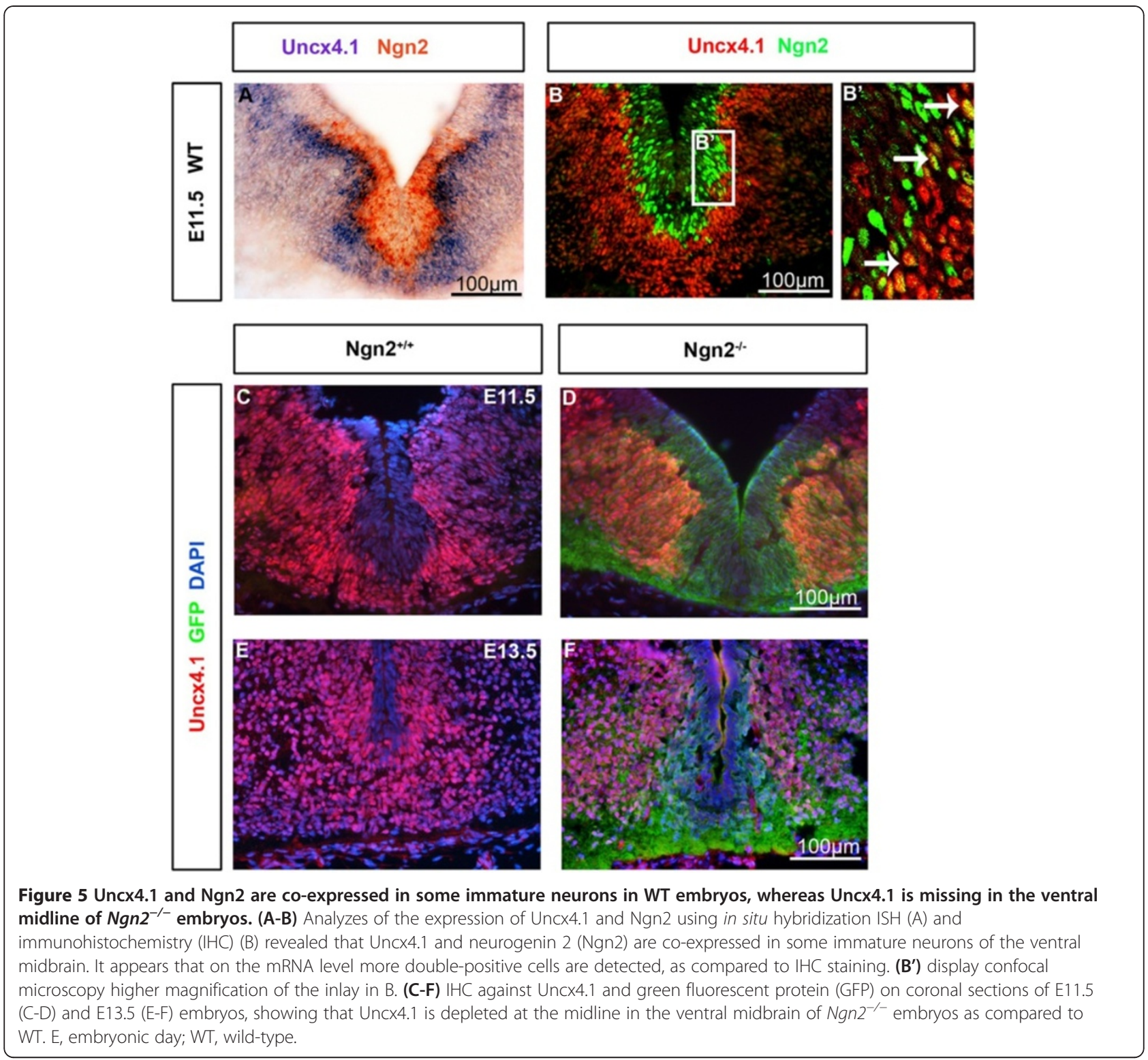

expression is mediated by an unknown factor that interacts with Uncx4.1.

\section{Normal development of midbrain GABAergic neurons in the absence of Uncx 4.1}

Our analysis indicated that the loss of Uncx4.1 is accompanied by downregulation of $N g n 2$ expression, resulting in a partial depletion of $\mathrm{mDA}$, without affecting cell proliferation or cell death. We therefore asked the question whether a cell identity switch might have occurred in the Uncx4.1-deficient midbrain and if these cells now ectopically express GABAergic neuron markers. Kala et al. showed that GABAergic neurons are located in the VTA-SNpr in late development [28]. To investigate whether mDA neurons switch their neuronal identity into a GABAergic destiny in the absence of Uncx4.1, or whether ectopic expression of GABAergic markers occurs in the mDA domain, we studied the expression of Helt and Mash1 which are essential for development of midbrain GABAergic neurons [17,29,30]. The expression of Helt (Figure 6, A-B) and Mash1 (Figure 4, G-H) was unaltered in the absence of Uncx4.1. To exclude the possibility that Uncx4.1 influences GABAergic neuron development postmitotically, we analyzed expression of Gad67 and Olig2 in Uncx4.1-deficient embryos. While Olig2 is confined to a specific subgroup of GABAergic neurons in the mantle layer Gad67 is found in all these neurons $[17,28]$. We did not observe a difference in the expression of Gad67 or Olig2 at early and late developmental stages (Figure 6, C-J). Together, these data 

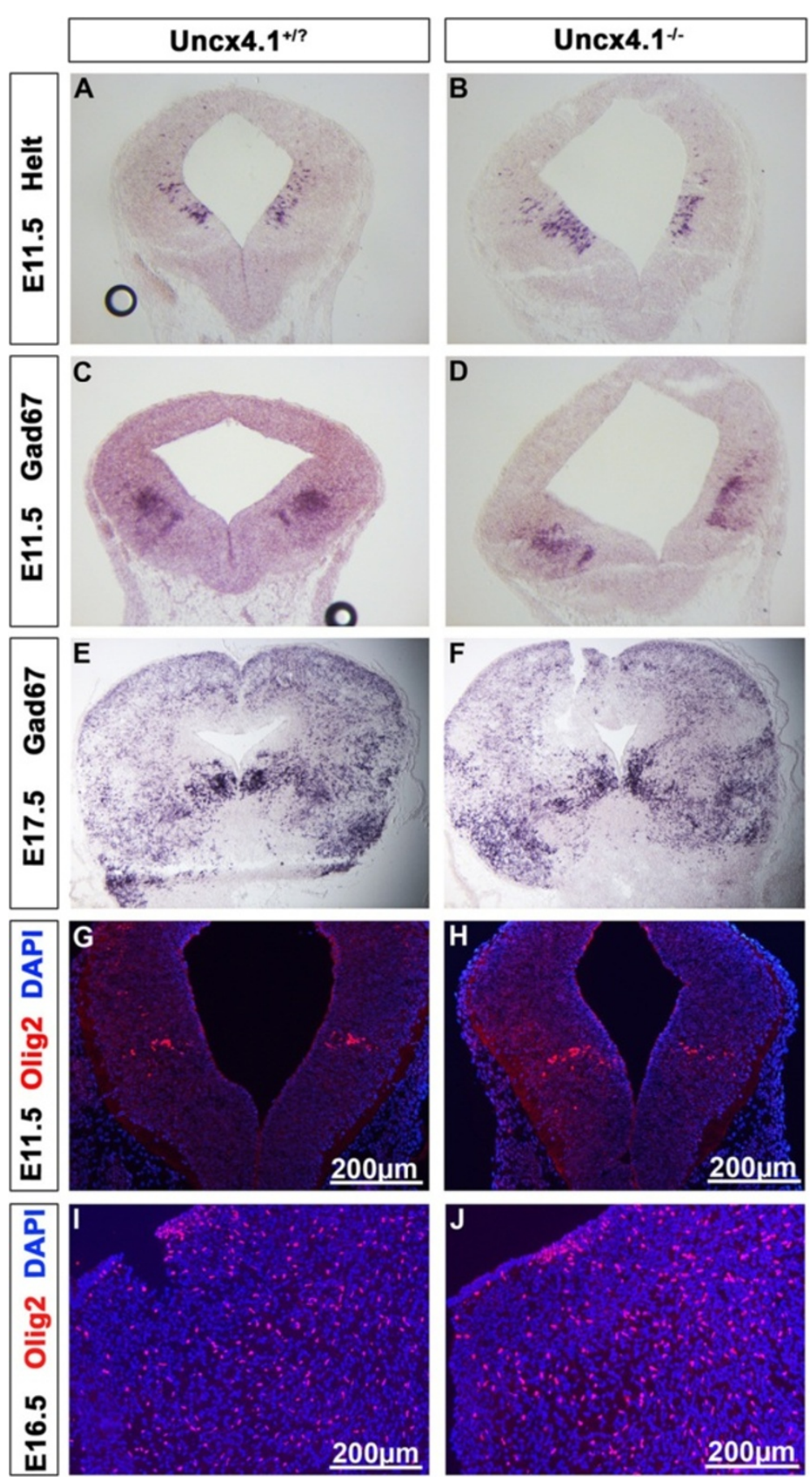

Figure 6 Midbrain GABAergic neurons are not affected in Uncx4.1-deficient embryos. (A-B) In situ hybridization (ISH) of Helt on E11.5 coronal sections reveals no difference in the absence of Uncx4.1. (C-F) The GABAergic marker GAD67 is expressed normally in Uncx4.1 $1^{-1-}$ embryos at E11.5 (C-D) and E17.5 (E-F) compared to control animals. (G-J) Immunohistochemistry (IHC) against Olig2 shows no difference in control and Uncx4.1 $1^{-/-}$embryos at E11.5 (G-H) and E16.5 (I-J). E, embryonic day.

demonstrated that inactivation of Uncx4.1 does not result in the ectopic activation of GABAergic neuron markers in the mDA domain. Therefore, we next examined glutamatergic neuron development in Uncx4.1-deficient embryos.
Increased numbers of Pax6-positive glutamatergic neurons in the ventral midbrain upon Uncx4.1 deficiency Glutamatergic neurons are located in the embryonic dorsal midbrain as well as in the ventral midbrain, and adjacent to the mDA neuron domain $[17,28,31]$. Brn3a 
was used as a marker for glutamatergic red nucleus neurons [32] that are located adjacent to the $\mathrm{mDA}$ neuron domain $[17,28,33]$. Using in situ hybridization (ISH) we observed an upregulation in the expression of $\mathrm{Brn} 3 \mathrm{a}$ in the ventral but not in the dorsal midbrain at all stages analyzed from E11.5 to E13.5 (Figure 7, A-B, and data not shown). Recently, it was reported that Nkx6.1 plays a crucial role for proper development of Brn3a-positive red nucleus neurons [18]. Applying IHC no apparent difference in Nkx6.1 expression at E12.5 (Figure 7 C-D) or

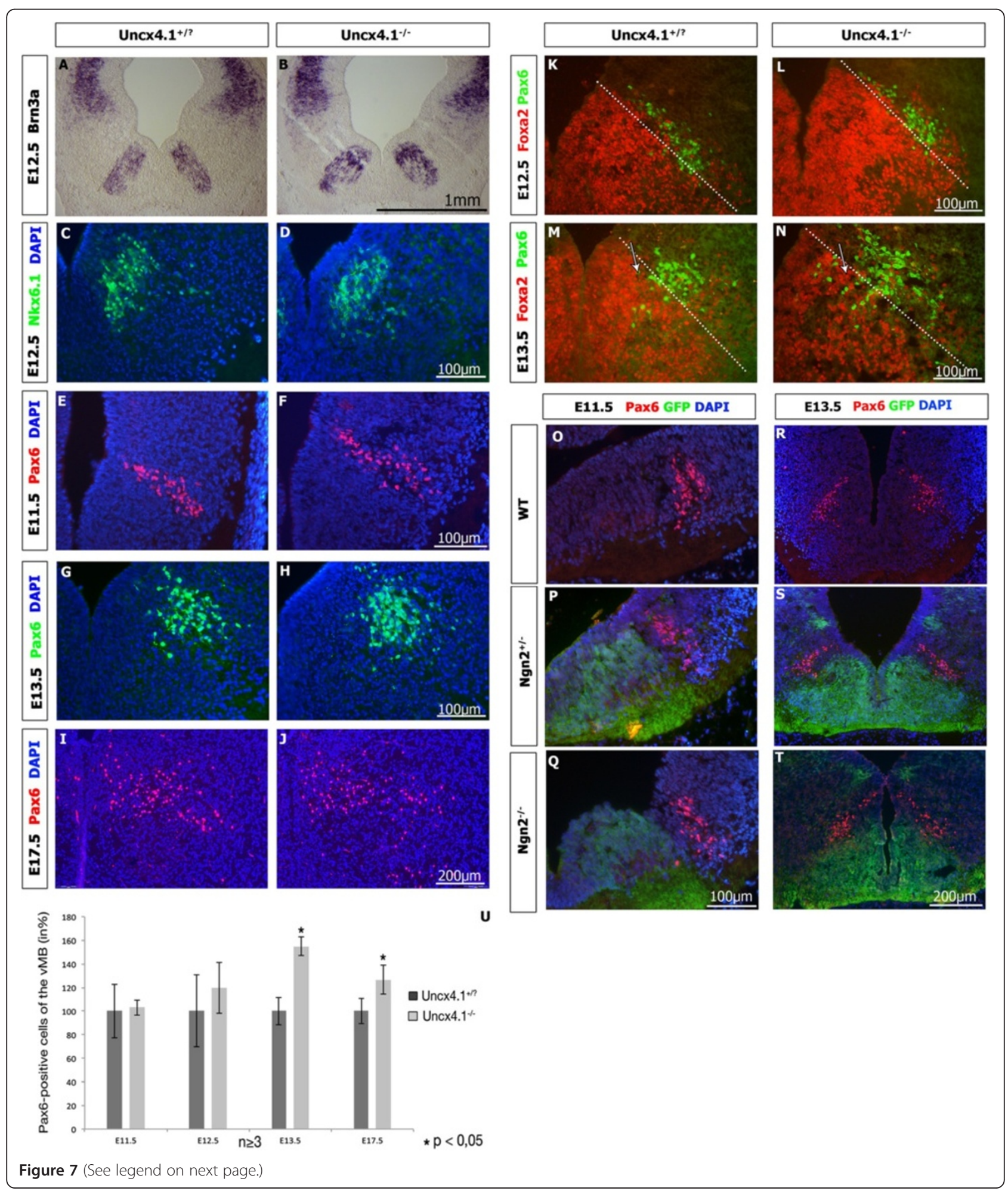


(See figure on previous page.)

Figure 7 Analysis of midbrain glutamatergic markers. (A-B) Brn3a expression is increased in the ventral midbrain of Uncx4.1 ${ }^{-/-}$embryos compared to controls. (C-D) Nkx6.1 expression is normal in E12.5 embryos, indicating a proper establishment of glutamatergic progenitors. (E-F) and (K-L) The numbers of Pax6-expressing cells are normal at E11.5 and E12.5 in Uncx4.1-deficient embryos compared to control. (G-J) and (M-N) Immunohistochemistry (IHC) reveals increased numbers of Pax6-expressing cells at E13.5 and E17.5 in the absence of Uncx4.1. (U) Quantification of Pax6-expressing cells in the ventral midbrain of Uncx4.1 $1^{-1-}$ and control embryos. Error bars represent the standard deviation. $P$ values $<0.05$ were assessed as statistically significant. (M-N) Co-labeling with Foxa2 revealed that Pax6 cells are extended more ventrally at E13.5 (white arrows). The dashed line marks the border in the Foxa2 expression domain. (O-T) Pax6 and green fluorescent protein (GFP) staining of $\mathrm{Ngn}^{-1-}$ embryos shows no difference in the numbers of Pax6-expressing cells in the basal plate of the ventral midbrain at E11.5 (O-Q) and E13.5 $(R-T)$. E, embryonic day; vMB: ventral midbrain.

E13.5 (data not shown) was detected. Interestingly, all Pax6-positive cells of the ventral midbrain are glutamatergic [28]. Using IHC Pax6 expression was not affected at E11.5, and E12.5 in mutant embryos (Figure 7E-F, U). However, starting with E13.5 the number of Pax6expressing cells was increased by $55 \%$ (Figure 7G-H, U) and by around $26 \%$ at E17.5 (Figure 7I-J, U). Double IHC against Pax6 and Foxa2 revealed, unlike at E12.5, that the Pax6 expression domain was slightly extended ventrally at E13.5 (Figure 7K-N). Further analysis using ISH and IHC indicated that the expression of Uncx4.1 is not affected in the midbrain of Pax6-deficient embryos at E11.5 and E17.5 (data not shown). We then asked whether the observed upregulation of Pax6 expression in Uncx4.1-deficient midbrain is caused by the diminution of Ngn2 transcription in Uncx4.1 mutant embryos. However, Pax6 expression was not modified in the basal plate of $N g n 2^{-/-}$embryos at all analyzed stages, indicating that the increase of Pax6-positive cells in Uncx4.1 $1^{-/-}$embryos may not be directly related to the reduction of $N g n 2$ (Figure 7O-T). In none of the above realized experiments we noted ectopic expression of glutamatergic markers in the $\mathrm{mDA}$ domain, indicating that a fate switch to a glutamatergic identity had not occurred. Our findings demonstrate that Uncx4.1 may play a critical role in the development of glutamatergic neuron subgroups in the ventral midbrain, and that the increased numbers of Pax6-positive neurons are not directly related to the partial depletion of $\mathrm{Ngn} 2$.

\section{Conditional inactivation of Uncx4.1 leads to a partial loss of mDA neurons in the SN of adult mutant mice}

Since Uncx4.1-deficient mice die shortly after birth $[12,13]$, we created a conditional Uncx4.1 mutant in order to examine mDA neurons in the adult midbrain. This conditional Cre-ER:Uncx4.1 mutant was obtained by crossing the floxed Uncx4.1 knockout line with an inducible Cre-ER mouse line [34] and injected tamoxifen at E12.5. Cre recombination efficiency was analyzed on P0 brains using an Uncx4.1 antibody. Around 90\% of the Uncx4.1 signal was lost in Cre-ER:Uncx4.1 ${ }^{\mathrm{del} /-}$ animals. Under such tamoxifen application conditions, and as reported for the Cre-ER [34], we assume that at
E14.5, 90\% of Uncx4.1 gene activity is lost. To analyze mDA neurons in adult Cre-ER:Uncx4.1 ${ }^{\text {del/- }}$ mice we performed IHC against TH and DAT. Such mutants exhibited a weak, but statistically not significant reduction in TH-positive cells compared to control animals $(n=3)$. However, the most lateral neurons of the SNpc always appeared reduced in mutant as compared to control midbrain (Figure 8, A-D, grey arrows and Additional file 3: Figure S3). Since almost all mDA precursors have been generated before E12.5 when tamoxifen was injected, but differentiation into mature neurons and migration still takes place, our findings suggest that Uncx4.1 might be involved in maturation and/or cell migration, but not in the generation of mDA neurons.

\section{Discussion}

In recent years enormous efforts were made to identify and characterize transcription factors controlling $\mathrm{mDA}$ neurogenesis, including Msx1 [3], Foxa2 [4], Lmx1a [3], Ngn2 [1,2], Nurr1 [6,35], En1/2 [36], Lmx1b [37] and Pitx3 $[19,21,38,39]$. In the transition from $\mathrm{VZ} \mathrm{mDA}$

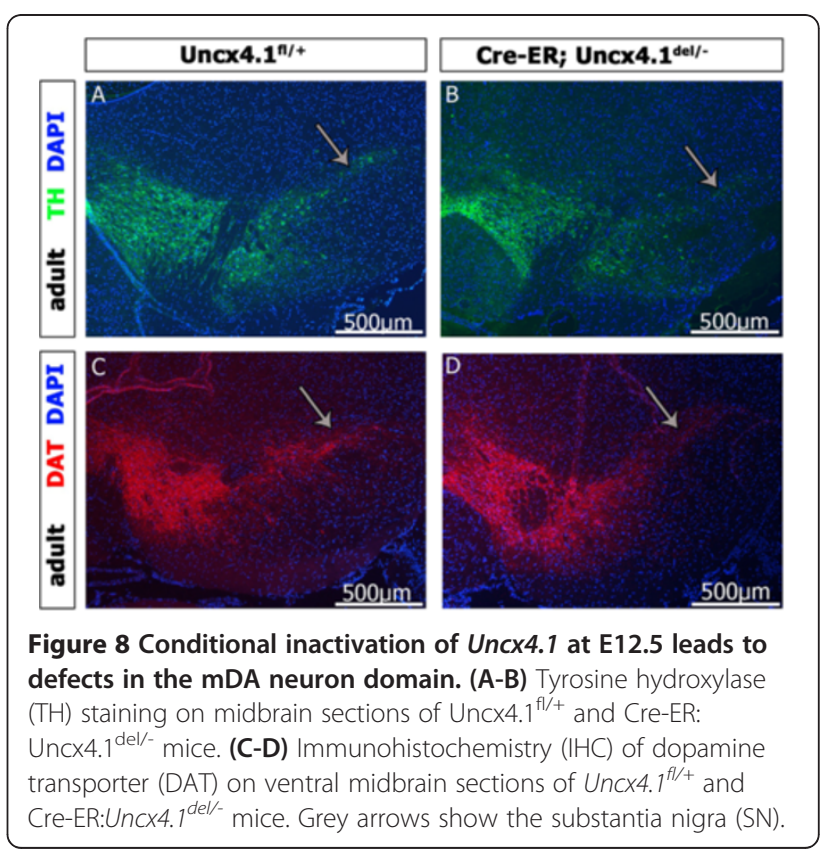


progenitors via precursors to mature mDA neurons, these factors provide a complex regulatory network required for full expansion of the heterogeneous mDAergic field of the midbrain [40]. In this study we provide evidence for an important novel role of the transcription factor Uncx4.1 in mDA neuron development. This role is exerted and confined in the critical phase of transition from progenitors to mature mDA neurons, roughly between E9.0 to E12.5, and it engages the proneural gene Ngn2. During this temporal window Uncx4.1 is required to sustain the expression of Ngn2. This suggests that Uncx4.1 is mainly a differentiation factor that may affect essential properties of $\mathrm{mDA}$ neurons in this period. For instance, our analysis suggests a possible implication of Uncx4.1 in the migration process of $\mathrm{mDA}$ precursors. In this respect it is important to note that Uncx4.1 deficiency causes a connectivity defect in the pituitary complex [14]. In this study we found that hypothalamic magnocellular neurons, neuroendocrine cells in the hypothalamus, fail to fully innervate the posterior pituitary, and instead run through the anterior pituitary. This points to an axon guidance defect in this neuronal system.

The current study also demonstrates that midbrain expression of Uncx4.1 is not exclusive for mDA neurons. It also includes GABAergic and glutamatergic neurons. We show that the loss of Uncx4.1 provokes an increase in the numbers of glutamatergic Pax6-marked cells in the ventral midbrain, suggesting an additional important function for Uncx4.1 activity in glutamatergic neurogenesis.

\section{The expression of Uncx4.1 in mDA neurons}

Developing midbrain dopaminergic neurons can be subdivided into three different groups. They arise from proliferating committed neural stem cells, first as postmitotic progenitors, then postmitotic precursors, and finally mature mDA neurons. Mature mDA neurons express the enzyme $\mathrm{TH}$ that is involved in the dopamine biosynthesis. In this study we could show that Uncx4.1 is expressed in nearly all TH-positive cells at E11.5 indicating that it is present in mature $\mathrm{mDA}$ neurons. Uncx4.1 expression seems to be induced in $\mathrm{mDA}$ progenitors that express Ngn2 [1,2]. This timing resembles the expression of Nurr1, a central transcription factor in the cell-specific expression and regulation of genes in mDA neurons [41]. In the whole midbrain, Uncx4.1 is absent from the VZ, but expressed in postmitotic neurons. Using IHC and ISH, we further demonstrate that Uncx4.1 is not restricted to a specific lineage but that it is present in most neuronal subgroups during midbrain development. This finding is consistent with a recent report demonstrating Uncx4.1 expression in all neuronal lineages of the olfactory bulb [15]. In conclusion, our findings support the idea that this transcription factor might have a general and cell-type independent role during neuronal development [15]. Furthermore, it may also promote particular properties of specific neuronal type, such as mDA neurons.

\section{The potential role of Uncx4.1 in mDA neuron development}

Our analysis of Uncx4.1 $1^{-/-}$embryos revealed a reduced expression of several mDA neuron markers, and including TH, DAT, Nurr1, and Pitx3. In addition, the numbers of Calbindin- and Calretinin-marked cells were severely decreased in this context. Calbindin and Calretinin are preferentially expressed in VTA neurons [23,24] whereas Calbindin labels mDA neurons of the VTA $[2,42]$. Therefore, the more pronounced reduction of Calbindin- and Calretinin-marked neurons indicates, that VTA neurons are possibly more affected by the absence of Uncx4.1 than those of the SN. This finding was further corroborated by the reduced expression of Otx2, a transcription factor expressed in mature mDA neurons of the VTA but not of the SN at E17.5 embryos and adult mice [25]. Although our findings indicate that the mDA neurons of the VTA are more affected than those of the $\mathrm{SN}$, we could also observe reduced numbers of $\mathrm{mDA}$ neurons in the SN. However, the minor decrease of mDA neurons in Uncx4.1-deficient mice is consistent with the notion that this factor only partially affects the differentiation of mDA neurons. Moreover, it appears that, although decreased, the level of Ngn2 expression is still sufficient for the generation DA neurons. It is also reasonable to assume that the loss of Uncx4.1 is compensated by another factor. It was shown in C. elegans that Unc-4, a homolog of Uncx4.1 $[10,43]$, acts together with a co-repressor and that these proteins are involved in the correct specification of the transmitter phenotype [22]. The persistent lower numbers of TH-positive neurons in Uncx4.1 mutants at E11.5, as well as at E17.5 of development, are consistent with the idea that the absence of Uncx4.1 does not provoke a delay in $\mathrm{mDA}$ neuron generation. In contrast to what has been described for the olfactory bulb of Uncx $4.1^{-/-}$mice, where cell death was reported [15], the lack of Uncx4.1 in the midbrain is not accompanied by an alteration in proliferation or apoptosis. This may suggest that in Uncx4.1-deficient mice the loss of a subpopulation of dopaminergic neurons is compensated by the accumulation of another neuronal cell type, which will be discussed below. Since Uncx4.1 is not expressed in the VZ where cellular specification occurs, and in addition, the majority of Uncx4.1-positive cells in the ventral midbrain are co-positive for $\mathrm{TH}$ at E11.5, it is more likely that Uncx4.1 acts during differentiation and not during specification of mDA neurons. The absence of Uncx 4.1 from $\mathrm{TH}$-positive cells at later stages (E17.5) supports 
the idea that Uncx4.1 is primarily acting during a short, critical window of differentiation of $\mathrm{mDA}$ neurons. Accordingly, the numbers of $\mathrm{mDA}$ neurons is unaffected at adult stages when Uncx4.1 was conditionally inactivated at E12.5, although it seems that the neurons of the $\mathrm{SN}$ are reduced. After their generation, mDA neurons first migrate along the dorsal ventral axis before they migrate laterally to their final destination. Since we could not recognize a difference in the cell content of $\mathrm{mDA}$ neurons in the conditional mutant mice, after deletion of Uncx4.1 at E12.5, but a reduction in the SN, this may suggest that Uncx4.1 is involved in the proper migration of these neurons. This is consistent with an earlier finding, where we could show that hypothalamic magnocellular neurons fail to fully innervate the posterior pituitary, and instead they connect to the anterior pituitary [14]. Moreover, it was shown that Unc-4 is involved in the correct establishment of the innervation of $\mathrm{VB}$ motor neurons in C.elegans [44-46]. This favors the hypothesis that Uncx4.1 is possibly implicated in the correct axonal guidance of $\mathrm{mDA}$ neurons and that the migration of mDA neurons to the $\mathrm{SN}$ is altered. The proneural gene $N g n 2$ is required for neuronal differentiation of mDA progenitors and starts to be expressed around E10.75 [1,2]. Within the mDA neuron population Ngn2 is exclusively present in dopaminergic neuron progenitors and immature neurons. In the absence of Uncx4.1, Ngn2 expression is down-regulated, providing additional evidence that the observed alterations in mDA neurons in Uncx4.1 mutant may be related to a differentiation defect in the progenitor or immature neuron pool. The transcription factor Nurr1 is expressed in immature and mature neurons and found essential for their maintenance $[6,47,48]$. It has been shown that Nurr1-positive cells are nearly absent in Ngn2-deficient embryos at E11.5 and that they recover around E13.5 [1]. In contrast to Ngn2 mutants, we did not observe such a recovery of Nurr1-positive cells in Uncx4.1-deficient mice. This is possibly due to the unaltered expression of Mash1 in the ventral midbrain [1]. Interestingly, we could not detect Uncx4.1 expression in the ventral midline of Ngn2-deficient midbrain. However, this may simply be explained by the nonestablished postmitotic neurons within the mDA domain of $N g n 2$ mutant at the analyzed time points. The Co-IP experiment and the fact that no binding sites for Uncx4.1 were identified on the Ngn2 locus provide evidence that Uncx4.1 and Ngn2 do not interact with each other. Therefore it is reasonable to assume that another unknown factor may be involved in the partial loss of mDA neurons provoked in Unc $x 4.1$ mutant embryos, and that the downregulation of $\mathrm{Ngn} 2$ is indirectly related to the moderate decrease of this neuron population. Overall, our results point to a mechanism of Uncx4-mediated differentiation events in the critical E9.0 to 12.5 time window, and indirectly engaging $N g n 2$.

\section{The potential role of Uncx4.1 in midbrain glutamatergic neuron development}

As mentioned above the loss of a subpopulation of $\mathrm{mDA}$ neurons may be compensated by the accumulation of another neuronal cell type. In fact, we could not detect ectopic expression of glutamatergic neuron markers in the mDA domain, which makes it unlikely that mDA neurons change their cell fate. However, we found increased Brn3amRNA signal in the ventral midbrain leading to the idea that Uncx4.1 acts on glutamatergic neuron development. The increase in the numbers of Pax6-positive neurons at E13.5 in Uncx4.1-deficient mice favors this hypothesis. The transcription factor Brn3a is expressed in glutamatergic red nucleus neurons, which are located adjacent to the mDA domain at E11.5 [17,28,31,32,49,50]. Since Nkx6.1 is required for the proper development of Brn3a-positive red nucleus neurons [18], the unaltered numbers of Nkx6.1positive cells indicate that the ventral glutamatergic progenitors are normally established. During development, the transcription factor Pax6 is expressed in a subset of glutamatergic neurons [28]. In this study, we demonstrate that the number of Pax6-expressing cells is increased in Unc $4.1^{-/-}$embryos starting with E13.5 and this alteration persists until at least E17.5. The increase in Pax6-positive cell number is not related to the downregulation of $\mathrm{Ngn} 2$ transcription. This is corroborated by the nonaltered expression of Pax6 in $N g n 2^{-/-}$mutant mice. Taken together, our findings suggest that Uncx4.1 acts on glutamatergic neuron differentiation. In addition, the normal expression of Uncx4.1 in Pax6-deficient midbrain at E11.5 may indicate that Uncx4.1 acts upstream of Pax6. Interestingly Ngn2-labeled glutamatergic neurons are more reduced in the dorsal than in the ventral midbrain of Uncx4.1 $1^{-/-}$ embryos. In this context, it was shown that Ngn2 promotes the glutamatergic neurotransmitter fate by repressing the GABAergic cell fate in the mouse cortex [51]. However, we did not observe any difference in GABAergic marker expression in Uncx4.1-deficient mice, suggesting that the remaining low level of $N g n 2$ is still sufficient to mediate glutamatergic over GABAergic neuron development. This finding is supported by the unaltered expression of the glutamatergic neuron marker Brn3a in the dorsal midbrain. Another explanation for the normal expression of GABAergic markers might be that Ngn1 compensate for the loss of Ngn2. Alternatively, Ngn2 itself may not be necessary for the development of a mesencephalic glutamatergic cell fate. Nakatani et al. (2007) showed in gain-offunction studies, that Ngn1 promotes the glutamatergic phenotype. Overall our results demonstrate that Uncx4.1 is required for the proper development of midbrain glutamatergic neurons. 


\section{Conclusions}

In this study, we have shown that the transcription factor Uncx4.1 is expressed in GABAergic, glutamatergic and dopaminergic neurons of the developing midbrain. A detailed analysis of the neuron population in the ventral midbrain of Uncx $4.1^{-/-}$embryos revealed a significant reduction in the content of mDA neurons. This study provides evidence that Uncx4.1 is a novel player in the complex regulatory network of $\mathrm{mDA}$ neuron differentiation and that it plays a role in the transition phase from progenitors to mature mDA neurons. Furthermore, our study uncovers that the loss of Uncx 4.1 provokes an increase in Pax6-positive neurons in the ventral midbrain, suggesting another important function for Uncx4.1 in midbrain glutamatergic neurogenesis.

\section{Methods}

\section{Animals}

Animal experimentation and housing was performed in agreement with the regulations of the animal welfare law of Germany and approved by LAVES Institution of Low Saxony (Approval Nr.:33.9- ${ }^{\circ} \odot--4250204--^{\circ} \odot--11 / 0402$ ).

Generation and genotyping of Uncx4.1.1- [12],

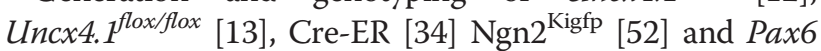
${ }_{-/-}$[53] mice has been described previously. Ngn2 $2^{\text {Kigfp/Kigfp }}$ are referred as $\mathrm{Ngn}^{-/-}$in this article. Either wild-type animals or Uncx4.1-heterozygous animals were used as control for comparison with Uncx4.1 $1^{-/-}$embryos. Such controls are shown as $U n c x 4.1^{+ \text {? }}$. All mice were kept on B6N background.

For conditional ablation of Uncx4.1 at E12.5 Uncx4.1 heterozygous mice were first crossed with Cre-ER mice to obtain Cre-ER:Uncx4.1 $1^{+/-}$mice. These mice were further crossed to $U n c x 4.1^{f l / f l}$ mice. $1 \mathrm{mg} / 10 \mathrm{~g}$ body weight tamoxifen was injected to the pregnant female at E12.5. The morning of the vaginal plug was considered as E0.5.

\section{Immunohistochemistry, in situ hybridization and X-gal staining}

Embryos were taken at the desired time point and used for in situ hybridization (ISH) or immunohistochemistry (ICH). Dissected brains or heads were placed in 4\% PFA for 1 to 16 hours according to their size. After fixation, embryos were washed three times for ten minutes in $1 \mathrm{x}$ PBS and, in case of cryoembedding followed by cryoprotection, in 30\% sucrose (in $1 \times \mathrm{PBS}$ ), 30 minutes in 50\% tissue freezing medium (Jung; Leica, Nussloch, Germany) in 30\% sucrose and embedded in tissue-freezing medium. In case of paraffin embedding, the tissue was dehydrated after washing in $1 \times$ PBS through ascending ethanol series before it was transferred in isopropanol/toluene (through an ascending isopropanol/toluene series) and finally embedded in paraffin.
ISH using digoxigenin-labeled single-stranded RNA probes was performed on $18 \mu \mathrm{m}$ thick cryosections according to Moorman et al. [54]. For two-colored ISH Dig- and Fluorescein-labeled probes were used. A detailed protocol for two-colored ISH is available upon request. The following in situ probes were used: Ngn2, Helt, Mash1, Uncx4.1, Gad67, Lmx1a, Nkx2.2, Nkx6.1 and Brn3a.

For IHC, $10 \mu \mathrm{m}$ thick cryo or paraffin sections were used. Paraffin sections were hydrated through descending ethanol series before boiling for one minute in unmasking solution (1:100 in water, Vector Laboratories, Burlingame, CA, USA). Afterwards, the sections were placed three times for five minutes in $1 \times$ PBS prior to blocking (in 10\% FCS $+0.01 \%$ Triton or $1 \%$ BSA $+0.01 \%$ Triton in PBS, sterile filtered). Primary antibodies were incubated overnight or for 72 hours at $4{ }^{\circ} \mathrm{C}$ in blocking solution. Secondary antibodies were diluted 1:750 in blocking solution and incubated for 70 minutes at room temperature. After secondary antibody sections were rinsed three times in $1 \mathrm{x}$ PBS before mounting in Vectrashield with DAPI (Vector Laboratories). Cryosections were processed the same way without descending ethanol series and boiling.

Primary antibodies used were rabbit anti-Uncx4.1 (1:750), goat anti-Hnf3ß (1:150, Santa Cruz Biotechnology, Santa Cruz, CA, USA), rabbit anti-TH (1:300, Chemicon, Billerica, MA, USA), mouse anti-TH (1:5000, SigmaAldrich, St. Louis, MO, USA), rat anti-DAT (1:100, Santa Cruz Biotechnology), rabbit anti-Pitx3 (1:1000), goat antiNurr1 (1:100, R\&D Systems, Minneapolis, MN, USA), rabbit anti-Pax6 (1:300, Covance, Princeton, NJ, USA), mouse anti-Pax6 (1:100, DSHB, Iowa City, IA, USA), rabbit anti-Nkx6.1 (1:3000 [55]), mouse anti-Nkx6.1 (1:100, DSHB), rabbit anti-Calbindin (1:200, Swant, Bellinzona, Switzerland), rabbit anti-Calretinin (1:300, SigmaAldrich), chicken anti-GFP (1:500, Abcam, Cambridge, MA, USA), anti-BrdU (1:50, Sigma-Aldrich), mouse antiNgn2 (1:100, R\&D Systems) and rabbit anti-Olig2 (1:500, Chemicon). Secondary antibodies were either Alexa488or Alexa594-conjugated and raised against mouse, rabbit, rat, chicken or goat (Invitrogen, Carlsbad, CA, USA).

$\mathrm{X}$-gal staining was performed as describes previously [56].

Pictures were taken with an Olympus BX60 fluorescent microscope or with an Olympus SZX 12 fluorescent microscope (Olympus, Tokyo, Japan). Confocal pictures were taken with Leica TCS SP5 confocal microscope (Leica Microsystems, Germany). Pictures were processed with Adobe Photoshop (Version 10.0) by overlaying the pictures, adjusting brightness, contrast and size.

\section{BrdU labeling and TUNEL assay}

$100 \mu \mathrm{l} / 10 \mathrm{~g}$ bodyweight BrdU (15 mg/ml in PBS) was intraperitoneally injected into pregnant females at E10.5 or E14.5. In the case of the E10.5-day-old embryos, the 
animal was sacrificed 24 hours after BrdU injection. In the case of the E14.5-day-old embryos, the animal was sacrificed 45 minutes after the injection. Embryos were processed for embedding in cryo media, followed by IHC against anti-BrdU (1:100, Roche, Basil, Switzerland) or against mouse anti-BrdU (1:100, Roche) together with rabbit anti-TH (1:200, Chemicon). Apoptosis was detected using the TUNEL assay. The TUNEL Apoptosis detection kit (Millipore, Billerica, MA, USA) was used following the manufacturer's instructions.

\section{Cell counting and statistical analysis}

Cell counts were done on images of coronal sections or directly under the microscope along the rostral-caudal axis of the midbrain. For embryonic stages, every fourth and for adult stages every eighth section was counted and the average was calculated for at least three animals. The twotailed unpaired Student's $t$ test was applied on the averages. Statistical significance was considered if $P \leq 0.05$.

\section{Cell culture, Co-immunoprecipitation (Co-IP) and western blotting (WB)}

Hela cells were cultured in DMEM medium with $10 \%$ fetal calf serum (FCS). For Co-IP the cells were seeded into a $10 \mathrm{~cm}$ dish. When 80 to $90 \%$ confluent cells were transfected using Lipofectamine 2000 (Invitrogen) following the manufacturer's manual. During this process either DMEM or Opti-MEM serum-free medium was used. The next morning, the proteasome inhibitor MG132 (Sigma-Aldrich) was applied to the cells at a final concentration of $20 \mu \mathrm{m}$. Twenty-four hours after transfection the cells were harvested and Co-IP using FLAG tagged beads was performed according to the manufacturer's recommendation (Sigma-Aldrich). For Co-IP Uncx4.1-c-myc was co-transfected with Ngn2FLAG. Detailed information about the plasmids is available upon request. After performing the Co-IP the samples were applied on a $12 \%$ SDS gel. Afterwards, the proteins were transferred to a membrane overnight followed by 90 minutes blocking in $5 \%$ milk powder in PBT. After blocking, the first antibody was incubated overnight at $4{ }^{\circ} \mathrm{C}$. The next day, the membrane was washed three times in $1 \mathrm{x}$ PBT and the second antibody was applied for one hour at room temperature. After three times washing for 30 minutes in 1 x PBT the protein was detected using Super Signal West Pico and Super Signal West Femto kit (both Pierce, Rockford, IL, USA).

First antibodies used are rabbit anti-FLAG (1:1000 Sigma-Aldrich) and mouse anti-c-myc (1:500 Santa Cruz Technology). Secondary antibodies used are anti-rabbitHRP (1:10000 Dianova, Hamburg, Germany) and antimouse-HRP (10000 Dianova).

\section{Additional files}

\begin{abstract}
Additional file 1: Figure S1. Uncx4.1-deficient midbrain shows no difference in cell proliferation at E11.5 as compared to control. (A-B) Immunohistochemistry (IHC) against 5-bromo-2'-deoxyuridine (BrdU) on coronal sections of E11.5 embryos after 24 hours of BrdU injection. (C-D) $I H C$ against BrdU and tyrosine hydroxylase (TH) 24 hours after BrdU injection. BrdU was injected at E10.5 and the animals were sacrificed 24 hours later at E11.5. E, embryonic day.
\end{abstract}

Additional file 2: Figure S2. Co-immunoprecipitation (Co-IP) of Uncx4.1 and Ngn2 provide no evidence for an interaction between the two factors. Hela cells were co-transfected as indicated and the lysates were used for a Co-IP assay using Flag tagged beads. Western blot (WB) analysis was performed using anti-c-myc and anti-Flag antibody.

Additional file 3: Figure S3. Conditional inactivation of Uncx4.1 at E12.5 results in defects in the substantia nigra (SN) as documented by alterations in dopamine transporter (DAT) expression (A-H), and tyrosine hydroxylase $(\mathrm{TH})$ expression (I-P) in adult animals. The white arrows point to the region of the $\mathrm{SN}$, which is affected in the mutant animals.

\section{Abbreviations}

BrdU: 5-bromo-2'-deoxyuridine; Co-IP: Co-immunoprecipitation: DA (T): Dopamine (transporter); E: Embryonic day; GFP: Green fluorescent protein; IHC: Immunohistochemistry; ISH: in situ hybridization; mDA: Midbrain dopaminergic neurons; Ngn2: Neurogenin 2; PBS: Phosphate buffered saline; RRF: Retrorubal field; SN(pc): Substantia nigra (pars compacta); TH: Tyrosine hydroxylase; TUNEL: Terminal deoxynucleotidyl transferase dUTP nick end labeling; VTA: Ventral tegmental area; VZ: Ventricular zone; WT: Wild-type.

\section{Competing interests}

The authors declare that they have no competing interests.

\section{Authors' contributions}

TR designed the study, carried out most of the experiments and drafted the manuscript, SB produced the Uncx4.1 antibody, GG and BZ performed some of the ISH and IHC experiments, FV helped carry out the western blotting experiments, AK and ML provided the Uncx4.1 knockout ES cells for conditional $\mathrm{KO}$ as well as the Uncx4.1-c-myc tagged construct for protein expression, PB helped in conceiving the study and writing the manuscript. AM conceived the study, and participated in its design, coordination and helped to draft and write the manuscript. All authors read and approved the manuscript.

\section{Acknowledgements}

We would like to thank W. Wurst, A. Simeone, J. Guimera, H.H. Arnold and M. Goulding for in situ probes as well as P. Serup for the rabbit anti-Nkx6.1 antibody, and F. Guillemot for Ngn2 KO mice. We thank C. Heuchel, A. Kurth and the BTL team for excellent support with the mice. The excellent technical assistance of T. Schulz is highly appreciated. We would like to highly acknowledge the fruitful discussions with A. Stoykova. This work was supported by the DFG Research Center Molecular Physiology of the Brain (CMPB), the Max Planck Society and the Dr. Helmut Storz Stiftung.

\section{Author details}

${ }^{1}$ Department of Molecular Cell Biology, Max Planck Institute of Biophysical Chemistry, Am Fassberg 11, Goettingen 37077, Germany. ²DF Research Center for the Molecular Physiology of the Brain, CMPB, Humboldtallee 23, Goettingen 37073, Germany. ${ }^{3}$ Medizinische Hochschule Hannover, Institute of Molecular Biology, Carl Neuberg Strasse 1, Hannover 30625, Germany. ${ }^{4}$ Biotechnology Centre of Oslo, University of Oslo, Gaustadalleen 21, Oslo 0349, Norway. ${ }^{5}$ Rudolf Magnus Institute of Neuroscience, University Medical Center Utrecht, Department of Neuroscience and Pharmacology,

Universiteitsweg 100, Utrecht 3584 CG, The Netherlands. ${ }^{6}$ Department of Molecular Neurobiology, Max Planck Institute for Experimental Medicine, Hermann-Rein-Strasse 3, Goettingen 37075, Germany. 'Department of Clinical Neurophysiology, University of Goettingen, Robert-Koch-Strasse 40,

Göttingen 37075, Germany. ${ }^{8}$ Current affiliation: Department of Biomedical Genetics, University Medical Center Utrecht, Universiteitsweg 100, Utrecht 3584 CG, The Netherlands. 
Received: 7 June 2012 Accepted: 13 November 2012

Published: 8 December 2012

\section{References}

1. Kele J, Simplicio N, Ferri AL, Mira H, Guillemot F, Arenas E, Ang SL: Neurogenin 2 is required for the development of ventral midbrain dopaminergic neurons. Development 2006, 133:495-505.

2. Andersson $\mathrm{E}$, Jensen JB, Parmar M, Guillemot F, Bjorklund A: Development of the mesencephalic dopaminergic neuron system is compromised in the absence of neurogenin 2. Development 2006, 133:507-516.

3. Andersson E, Tryggvason U, Deng Q, Friling S, Alekseenko Z, Robert B, Perlmann T, Ericson J: Identification of intrinsic determinants of midbrain dopamine neurons. Cell 2006, 124:393-405.

4. Ferri AL, Lin W, Mavromatakis YE, Wang JC, Sasaki H, Whitsett JA, Ang SL: Foxa1 and Foxa2 regulate multiple phases of midbrain dopaminergic neuron development in a dosage-dependent manner. Development 2007, 134:2761-2769.

5. Mavromatakis YE, Lin W, Metzakopian E, Ferri AL, Yan CH, Sasaki H, Whisett J, Ang SL: Foxa1 and Foxa2 positively and negatively regulate Shh signalling to specify ventral midbrain progenitor identity. Mech Dev 2011 , 128:90-103.

6. Saucedo-Cardenas O, Quintana-Hau JD, Le WD, Smidt MP, Cox JJ, De Mayo F, Burbach JP, Conneely OM: Nurr1 is essential for the induction of the dopaminergic phenotype and the survival of ventral mesencephalic late dopaminergic precursor neurons. Proc Natl Acad Sci USA 1998, 95:40134018.

7. Puelles E, Annino A, Tuorto F, Usiello A, Acampora D, Czerny T, Brodski C, Ang SL, Wurst W, Simeone A: Otx2 regulates the extent, identity and fate of neuronal progenitor domains in the ventral midbrain. Development 2004, 131:2037-2048.

8. Tang M, Miyamoto $Y$, Huang EJ: Multiple roles of beta-catenin in controlling the neurogenic niche for midbrain dopamine neurons. Development 2009, 136:2027-2038.

9. Tang M, Villaescusa JC, Luo SX, Guitarte C, Lei S, Miyamoto Y, Taketo MM, Arenas $E_{\text {, Huang }} \mathrm{EJ}$ : Interactions of Wnt/beta-catenin signaling and sonic hedgehog regulate the neurogenesis of ventral midbrain dopamine neurons. J Neurosci 2010, 30:9280-9291.

10. Mansouri A, Yokota Y, Wehr R, Copeland NG, Jenkins NA, Gruss P: Pairedrelated murine homeobox gene expressed in the developing sclerotome, kidney, and nervous system. Dev Dyn 1997, 210:53-65.

11. Neidhardt LM, Kispert A, Hermann BG: A mouse gene of the paired-related homeobox class expressed in the caudal somite compartment and the developing vertebral column, kidney and nervous system. Dev Genes Evol 1997, 207:330-339.

12. Mansouri A, Voss AK, Thomas T, Yokota Y, Gruss P: Uncx4.1 is required for the formation of the pedicles and proximal ribs and acts upstream of Pax9. Development 2000, 127:2251-2258.

13. Leitges M, Neidhardt L, Haenig B, Herrmann BG, Kispert A: The paired homeobox gene Uncx4.1 specifies pedicles, transverse processes and proximal ribs of the vertebral column. Development 2000, 127:2259-2267.

14. Asbreuk $\mathrm{CH}$, van Doorninck JH, Mansouri A, Smidt MP, Burbach JP: Neurohypophysial dysmorphogenesis in mice lacking the homeobox gene Uncx4.1. J Mol Endocrinol 2006, 36:65-71.

15. Sammeta N, Hardin DL, McClintock TS: Uncx regulates proliferation of neural progenitor cells and neuronal survival in the olfactory epithelium. Mol Cell Neurosci 2010, 45:398-407.

16. Nakatani T, Mizuhara E, Minaki $Y$, Sakamoto $Y$, Ono $Y$ : Helt, a novel basichelix-loop-helix transcriptional repressor expressed in the developing central nervous system. J Biol Chem 2004, 279:16356-16367.

17. Nakatani T, Minaki Y, Kumai M, Ono Y: Helt determines GABAergic over glutamatergic neuronal fate by repressing $\mathrm{Ngn}$ genes in the developing mesencephalon. Development 2007, 134:2783-2793.

18. Prakash N, Puelles E, Freude K, Trumbach D, Omodei D, Di Salvio M, Sussel L, Ericson J, Sander M, Simeone A, Wurst W: Nkx6-1 controls the identity and fate of red nucleus and oculomotor neurons in the mouse midbrain. Development 2009, 136:2545-2555.

19. Smidt MP, van Schaick HS, Lanctot C, Tremblay JJ, Cox JJ, van der Kleij AA, Wolterink G, Drouin J, Burbach JP: A homeodomain gene Ptx3 has highly restricted brain expression in mesencephalic dopaminergic neurons. Proc Natl Acad Sci USA 1997, 94:13305-13310.
20. Smidt MP, Smits SM, Bouwmeester $H$, Hamers FP, van der Linden AJ, Hellemons AJ, Graw J, Burbach JP: Early developmental failure of substantia nigra dopamine neurons in mice lacking the homeodomain gene Pitx3. Development 2004, 131:1145-1155.

21. Maxwell SL, Ho HY, Kuehner E, Zhao S, Li M: Pitx3 regulates tyrosine hydroxylase expression in the substantia nigra and identifies a subgroup of mesencephalic dopaminergic progenitor neurons during mouse development. Dev Biol 2005, 282:467-479.

22. Von Stetina SE, Fox RM, Watkins KL, Starich TA, Shaw JE, Miller DM 3rd: UNC-4 represses $\mathrm{CEH}-12 / \mathrm{HB} 9$ to specify synaptic inputs to VA motor neurons in C. elegans. Genes Dev 2007, 21:332-346.

23. Nemoto C, Hida T, Arai R: Calretinin and calbindin-D28k in dopaminergic neurons of the rat midbrain: a triple-labeling immunohistochemical study. Brain Res 1999, 846:129-136.

24. Liang $C L$, Sinton $C M$, Sonsalla PK, German DC: Midbrain dopaminergic neurons in the mouse that contain calbindin-D28k exhibit reduced vulnerability to MPTP-induced neurodegeneration. Neurodegeneration 1996, 5:313-318.

25. Di Salvio M, Di Giovannantonio LG, Omodei D, Acampora D, Simeone A Otx2 expression is restricted to dopaminergic neurons of the ventral tegmental area in the adult brain. Int J Dev Biol 2010, 54:939-945.

26. Ono Y, Nakatani T, Sakamoto Y, Mizuhara E, Minaki Y, Kumai M, Hamaguch A, Nishimura M, Inoue $Y$, Hayashi H, Takahashi J, Imai T: Differences in neurogenic potential in floor plate cells along an anteroposterior location: midbrain dopaminergic neurons originate from mesencephalic floor plate cells. Development 2007, 134:3213-3225.

27. Yan CH, Levesque M, Claxton S, Johnson RL, Ang SL: Lmx1a and Imx1b function cooperatively to regulate proliferation, specification, and differentiation of midbrain dopaminergic progenitors. J Neurosci 2011 , 31:12413-12425.

28. Kala K, Haugas M, Lillevali K, Guimera J, Wurst W, Salminen M, Partanen J: Gata2 is a tissue-specific post-mitotic selector gene for midbrain GABAergic neurons. Development 2009, 136:253-262.

29. Miyoshi G, Bessho Y, Yamada S, Kageyama R: Identification of a novel basic helix-loop-helix gene, Heslike, and its role in GABAergic neurogenesis. J Neurosci 2004, 24:3672-3682.

30. Peltopuro $P$, Kala K, Partanen J: Distinct requirements for Ascl1 in subpopulations of midbrain GABAergic neurons. Dev Biol 2010, 343:63-70.

31. Waite MR, Skidmore JM, Billi AC, Martin JF, Martin DM: GABAergic and glutamatergic identities of developing midbrain Pitx2 neurons. Dev Dyn 2011, 240:333-346

32. Agarwala S, Ragsdale CW: A role for midbrain arcs in nucleogenesis. Development 2002, 129:5779-5788.

33. Agarwala S, Sanders TA, Ragsdale CW: Sonic hedgehog control of size and shape in midbrain pattern formation. Science 2001, 291:2147-2150.

34. Hayashi S, McMahon AP: Efficient recombination in diverse tissues by a tamoxifen-inducible form of Cre: a tool for temporally regulated gene activation/inactivation in the mouse. Dev Biol 2002, 244:305-318.

35. Smits SM, Ponnio T, Conneely OM, Burbach JP, Smidt MP: Involvement of Nurr1 in specifying the neurotransmitter identity of ventral midbrain dopaminergic neurons. Eur J Neurosci 2003, 18:1731-1738.

36. Simon HH, Saueressig H, Wurst W, Goulding MD, O'Leary DD: Fate of midbrain dopaminergic neurons controlled by the engrailed genes. J Neurosci 2001, 21:3126-3134

37. Smidt MP, Asbreuk CH, Cox JJ, Chen H, Johnson RL, Burbach JP: A second independent pathway for development of mesencephalic dopaminergic neurons requires Lmx1b. Nat Neurosci 2000, 3:337-341.

38. van den Munckhof P, Luk KC, Ste-Marie L, Montgomery J, Blanchet PJ, Sadikot AF, Drouin J: Pitx3 is required for motor activity and for survival of a subset of midbrain dopaminergic neurons. Development 2003 130:2535-2542.

39. Nunes I, Tovmasian LT, Silva RM, Burke RE, Goff SP: Pitx3 is required for development of substantia nigra dopaminergic neurons. Proc Natl Acad Sci USA 2003, 100:4245-4250.

40. Smidt MP, Burbach JP: How to make a mesodiencephalic dopaminergic neuron. Nat Rev Neurosci 2007, 8:21-32.

41. Jacobs FM, van Erp S, van der Linden AJ, von Oerthel L, Burbach JP, Smidt MP: Pitx3 potentiates Nurr1 in dopamine neuron terminal differentiation through release of SMRT-mediated repression. Development 2009, 136:531-540. 
42. Thompson L, Barraud P, Andersson E, Kirik D, Bjorklund A: Identification of dopaminergic neurons of nigral and ventral tegmental area subtypes in grafts of fetal ventral mesencephalon based on cell morphology, protein expression, and efferent projections. J Neurosci 2005, 25:6467-6477.

43. Rovescalli AC, Asoh S, Nirenberg M: Cloning and characterization of four murine homeobox genes. Proc Natl Acad Sci USA 1996, 93:10691-10696.

44. Miller DM, Shen MM, Shamu CE, Burglin TR, Ruvkun G, Dubois ML, Ghee M, Wilson L: C. elegans unc-4 gene encodes a homeodomain protein that determines the pattern of synaptic input to specific motor neurons. Nature 1992, 355:841-845.

45. White JG, Southgate E, Thomson JN: Mutations in the Caenorhabditis elegans unc-4 gene alter the synaptic input to ventral cord motor neurons. Nature 1992, 355:838-841.

46. Pflugrad A, Meir JY, Barnes TM, Miller DM 3rd: The Groucho-like transcription factor UNC-37 functions with the neural specificity gene unc-4 to govern motor neuron identity in C. elegans. Development 1997, 124:1699-1709.

47. Zetterstrom RH, Solomin L, Jansson L, Hoffer BJ, Olson L, Perlmann T: Dopamine neuron agenesis in Nurr1-deficient mice. Science 1997, 276:248-250.

48. Wallen AA, Castro DS, Zetterstrom RH, Karlen M, Olson L, Ericson J, Perlmann T: Orphan nuclear receptor Nurr1 is essential for Ret expression in midbrain dopamine neurons and in the brain stem. Mol Cell Neurosci 2001, 18:649-663.

49. McEvilly RJ, Erkman L, Luo L, Sawchenko PE, Ryan AF, Rosenfeld MG: Requirement for Brn-3.0 in differentiation and survival of sensory and motor neurons. Nature 1996, 384:574-577.

50. Xiang M, Gan L, Zhou L, Klein WH, Nathans J: Targeted deletion of the mouse POU domain gene Brn-3a causes selective loss of neurons in the brainstem and trigeminal ganglion, uncoordinated limb movement, and impaired suckling. Proc Natl Acad Sci USA 1996, 93:11950-11955.

51. Schuurmans C, Armant O, Nieto M, Stenman JM, Britz O, Klenin N, Brown C, Langevin LM, Seibt J, Tang H, Cunningham JM, Dyck R, Walsh C, Campbell $K$, Polleux F, Guillemot F: Sequential phases of cortical specification involve Neurogenin-dependent and -independent pathways. EMBO J 2004, 23:2892-2902.

52. Seibt J, Schuurmans C, Gradwhol G, Dehay C, Vanderhaeghen P, Guillemot F, Polleux F: Neurogenin2 specifies the connectivity of thalamic neurons by controlling axon responsiveness to intermediate target cues. Neuron 2003, 39:439-452.

53. St-Onge L, Sosa-Pineda B, Chowdhury K, Mansouri A, Gruss P: Pax6 is required for differentiation of glucagon-producing alpha-cells in mouse pancreas. Nature 1997, 387:406-409.

54. Moorman AF, Houweling AC, de Boer PA, Christoffels VM: Sensitive nonradioactive detection of mRNA in tissue sections: novel application of the whole-mount in situ hybridization protocol. J Histochem Cytochem 2001, 49:1-8.

55. Jensen J, Serup P, Karlsen C, Nielsen TF, Madsen OD: mRNA profiling of rat islet tumors reveals nkx 6.1 as a beta-cell-specific homeodomain transcription factor. J Biol Chem 1996, 271:18749-18758.

56. Zembrzycki A, Griesel G, Stoykova A, Mansouri A: Genetic interplay between the transcription factors Sp8 and Em×2 in the patterning of the forebrain. Neural Dev 2007, 2:8.

doi:10.1186/1749-8104-7-39

Cite this article as: Rabe et al:: The transcription factor Uncx4.1 acts in a short window of midbrain dopaminergic neuron differentiation. Neural Development 2012 7:39.

\section{Submit your next manuscript to BioMed Central and take full advantage of:}

- Convenient online submission

- Thorough peer review

- No space constraints or color figure charges

- Immediate publication on acceptance

- Inclusion in PubMed, CAS, Scopus and Google Scholar

- Research which is freely available for redistribution

Submit your manuscript at www.biomedcentral.com/submit 